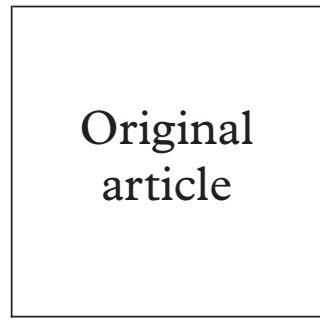

\title{
Penile granuloma annulare
}

\author{
N Narouz, P S Allan, A H Wade
}

A case of granuloma annulare (GA) localised to the shaft of the penis is reported with a brief review of the current literature. We concluded that penile GA, although rare, should be considered in the differential diagnosis of granulomatous lesions of the penis and that histopathological examination of the lesion is essential for the diagnosis.

(Sex Transm Inf 1999;75:186-187)

Keywords: penile granuloma annulare

\section{Introduction}

Penile granuloma annulare (GA) is an uncommon, benign, inflammatory, cutaneous lesion that has no proved aetiology ${ }^{1}$ or widely accepted pathogenesis. ${ }^{2}$ It usually involves the hands and feet, ${ }^{13-5}$ commonly occurs in young females, ${ }^{5}$ and is usually a self limiting condition. ${ }^{6}$ The disease consists of granulomatous inflammation and collagen alteration in the dermis of the involved site, ${ }^{2}$ which may be localised or generalised. Penile GA is not often recorded and, to the best of our knowledge, there have only been four previous reported cases. $^{7-10} \mathrm{~A}$ case of penile GA is reported with a review of literature.

\section{Case report}

A 23 year old white man presented to the genitourinary medicine clinic with a 1 month history of what he called "warts" on his penis. The lesion was neither painful nor pruritic and did not interfere with erection or intercourse. There was no other urogenital symptom. The patient denied any history of trauma or exposure to any toxic chemicals. He had no similar lesions before and there was no history of any previous STIs.

His medical and family histories were unremarkable. There was no known history of allergy and he was not taking any medication. 26 February 1999

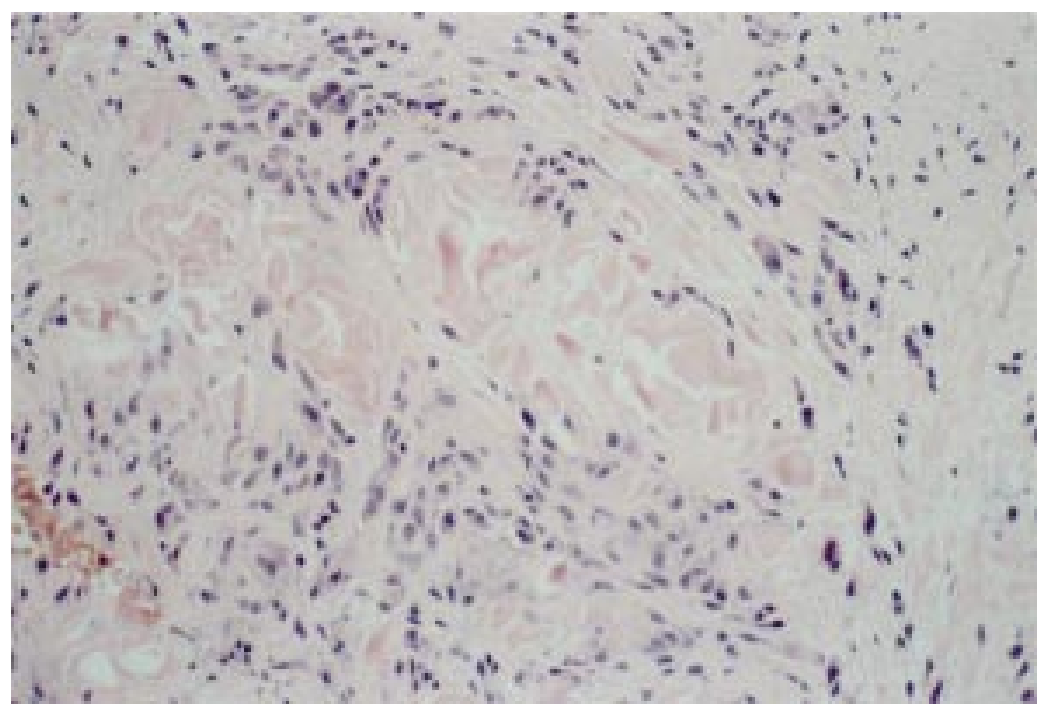

Figure 1 Localised degenerated collagen surrounded by inflammatory cells.
There was no history of injecting drug use although he smoked cannabis occasionally. The patient was heterosexual with no regular sexual relationship at the time of presentation.

On examination, a firm, smooth, pea sized nodule was noted at the middle of the dorsal aspect of the shaft of his penis. The lesion was not tender and the overlying skin was intact. There was no lymphadenopathy or any other abnormality on genital examination. General examination was unremarkable with no evidence of any other lesions elsewhere.

Cultures of swabs for gonorrhoea and chlamydia were reported as negative and syphilis serology tests (VDRL, TPHA) were also negative. Non-specific urethritis (NSU) was revealed by microscopic examination of the urethral swab and was treated with doxycycline. Urine examination by Dipstix revealed no abnormality. After counselling, HIV test was offered but the patient declined it.

The nodule was completely excised surgically, under local anaesthesia ( $1 \%$ lignocaine). The histological examination revealed a localised granulomatous reaction surrounding a central core of necrobiotic collagen. There was no evidence of malignancy. Special stains for fungi and acid fast bacilli were negative. The histological picture was consistent with that of granuloma annulare (fig 1). Follow up of the patient (12 months so far) revealed satisfactory healing of the wound with no evidence of recurrence or appearance of any other lesion.

\section{Discussion}

Granuloma annulare can express itself anywhere on the body, although the hands and feet are more frequently involved. It is more common in females than males $(2.3: 1) . .^{1-5} \mathrm{GA}$ can present in four different clinical manifestations-annular (localised), generalised (disseminated), nodular (subcutaneous), and perforating forms. Each type has its clinical characteristics. ${ }^{34}$ The annular (localised) GA is the most common form. ${ }^{11}$ Subcutaneous (nodular) GA usually presents as rapidly growing soft tissue firm nodules that are usually located on the extremities. The overlying epidermis appears normal. Subcutaneous nodules are usually solitary and can be associated with the annular form. GA lesions are typically asymptomatic, non-tender, and non-pruritic. 
In our case, the clinical picture was consistent with the typical nodular (subcutaneous) type of GA. The penis is a rare site for GA and in all of the reported cases of penile GA, including our case, the condition involved the penis only with no other GA lesions elsewhere in the body.

The aetiology of GA is unknown. ${ }^{1}$ A number of unproved aetiological factors have been suggested. These include primary necrobiosis, ultraviolet light, post-tuberculin skin tests, fungal infections, arthropod bites, contact with irritant material, thyroiditis, trauma, and viral infections (for example, HZV, HIV). ${ }^{4}$ Currently, GA has no established association with other genitourinary conditions, ${ }^{8}$ and the association between GA and diabetes mellitus or malignancy is not clear. ${ }^{1}$ In our case there was no history of any suggested aetiology.

Currently there are four possible hypotheses as to the pathogenesis of GA lesions. This may be a vasculitis leading to necrotising changes, trauma induced primary necrobiosis, monocytic release of lysosomal enzymes, or type IV hypersensitivity reaction. The most likely mechanism remains unclear. ${ }^{1}$

Histologically, GA is characterised by focal incomplete degeneration of collagen with reactive inflammation and fibrosis. The degenerated collagen is surrounded by palisading inflammatory cells. ${ }^{11}$ The cells are mostly histiocytes mixed with monocytes with few, if any, giant cells and variable numbers of lymphocytes and fibroblasts. These changes are almost always confined to the dermis with normal epidermis. The palisading seen histologically and the typical annular eruption seen clinically justify the term annulare.

Differential diagnosis of penile GA includes epithelial cysts, dermatofibroma, warts, syphilis, sarcoidosis, tuberculosis, ring worm, penile carcinoma, and epithelioid sarcoma. ${ }^{59}$

Laboratory tests are not particularly helpful in diagnosing GA directly. ${ }^{2}$ However, some investigations can help in the differential diagnosis (for example, erythrocyte sedimentation rate, serum glucose, antibody screen). ${ }^{12}$ In our case, there was no clinical evidence to warrant undertaking more laboratory tests.

Most cases of GA resolve spontaneously ( $75 \%$ within 2 years). ${ }^{6}$ However, patients may ask for treatment for cosmetic reasons. Recurrence at the original site is also common after resolution $(40 \%),{ }^{2}$ although these lesions tend to heal spontaneously more quickly than the original ones. ${ }^{4}$
The treatment used in this case was surgical excision, which was successful. Post treatment follow up of patients is important because of the tendency of the lesion to recur. ${ }^{7}$ Generally, a number of treatments have been used for GA, although the efficacy of a treatment is difficult to evaluate, in view of the tendency of the lesion to resolve spontaneously and to recur. Some of the suggested treatments with varying degrees of success include steroids (intralesional injection, topical, or systemic), destructive treatment (for example, cryotherapy, surgical excision), and systemic treatments (for example, steroids, antimalarials). ${ }^{42}$ Any type of trauma to the localised lesions, even the process of biopsy itself, may initiate resolution of the lesion.

Penile GA, although rare, should be considered in the differential diagnosis of granulomatous lesions of the penis. Diagnosis of GA depends upon clinical suspicion, biopsy, and histological examination. Histological examination of the lesion is crucial for its diagnosis. Surgical excision of the lesion is usually effective although recurrence may occur. Recent histochemical work, involving RNA probes for example, may prove to be valuable tools in the diagnosis of GA.

We would like to thank Dr David Snead, consultant histopathology at Walsgrave Hospital, Coventry, for providing us with the histopathological report and photograph.

1 Dabski K, Winkelmann RK. Generalised granuloma annulare: clinical and laboratory findings in 100 patients. $\mathcal{F} \mathrm{Am}$ Acad Dermatol 1989;20:39-47.

2 Smith MD, Downie JB, DiCostanzo D. Granuloma annulare. Int $\mathcal{F}$ Dermatol 1997;36:326-33.

$3 \mathrm{Zax}$ RH, Callen JP Granulomatous reactions. In: Sams WM, Lynch PJ, eds. Principles and practice of dermatology. New York: Churchill Livingstone, 1990:619-28.

4 Dahl MV. Granuloma annulare. In: Fitzpatrick TB, Eisen AZ, Wolff $\mathrm{K}$, et al, eds. Dermatology in general medicine. New York: McGraw-Hill, 1993:1187-91.

5 Cunliffe WJ. Necrobiotic disorders. In: Rook A, Wilkinson DS, Ebling FJG, Champion RH, Burton JL, eds. Text book of dermatology. 4th ed. Oxford: Blackwell Scientific, 1986:1687-90

6 Barron DF, Cootauco MH, Cohen BA. Granuloma annulare. A clinical review. Lippincotts Prim Care Pract 1997;1:33-9.

7 Kossard S, Collins AG, Wegman A, et al. Necrobiotic granulomas localised to the penis: a possible variant of subgranulomas localised to the penis: a possible variant of sub$101-4$

8 Hillman RJ, Waldron S, Walker MM, et al. Granuloma annulare of the penis. Genitourin Med 1992;68:47-9

9 Laird SM. Granuloma annulare of the penis. Genitourin Med 1992;68:277.

10 Trap R, Wiebe B. Granuloma annulare localised to the shaft of the penis. Scand F Urol Nephrol 1993;27:549-51.

1 Mallory SB. Infiltrative diseases. In Schachner LA, Hansen RC, eds. Paediatric dermatology 2nd ed. New York: Churchill Livingstone, 1995;2:834-6.

12 Miketa JP, Prigoff MM. Granuloma annulare: a case presentation of the typical and subcutaneous forms. F Foot Ankle Surg 1993;32:34-7. 